\title{
A PRIORI BOUNDS FOR BOUNDARY SETS ${ }^{1}$
}

\section{J. W. BEBERNES AND ROSS FRAKER}

Abstract. Consider $y^{\prime \prime}=f\left(t, y, y^{\prime}\right)$ with boundary conditions $\left(0, y(0), y^{\prime}(0)\right) \in S_{1},\left(1, y(1), y^{\prime}(1)\right) \in S_{2}$. It is shown that the boundary value problem has a solution for certain boundary sets $S_{1}$ and $S_{2}$ which depend on the assumed Nagumo condition for $f\left(t, y, y^{\prime}\right)$.

1. Consider the boundary value problem

$$
\begin{gathered}
y^{\prime \prime}=f\left(t, y, y^{\prime}\right), \\
\left(0, y(0), y^{\prime}(0)\right) \in S_{1}, \quad\left(1, y(1), y^{\prime}(1)\right) \in S_{2},
\end{gathered}
$$

where $f(t, y, z)$ is continuous on $[0,1] \times R^{2}$ and the boundary sets $S_{1}$ and $S_{2}$ are subsets of $[0,1] \times R^{2}$ which will be defined later.

The first author together with $\mathrm{R}$. Wilhelmsen has previously considered boundary value problem BVP (1)-(2) in [1], [2], and [3]. Jackson and Klaasen [5] and Sesdziwy [7] have also investigated BVP (1)-(2). Assuming $f(t, y, z)$ satisfies a Nagumo condition, i.e., a growth restriction in the $z$-variable, when $(t, y)$ lies in some compact set, a priori bounds can be determined which restrict the growth of the derivative $y^{\prime}(t)$ of any solution $y(t)$ of (1) as long as $(t, y(t))$ lies in the given compact set. The purpose of this paper is to show how these a priori bounds can be utilized to determine $S_{1}$ and $S_{2}$. These a priori bounds permit the construction of boundary sets, more general than hereto considered, for which BVP (1)-(2) has at least one solution.

2. Let $\alpha(t), \beta(t) \in C[0,1]$ with $\alpha(t) \leqq \beta(t)$ for all $t \in[0,1]$. The function $f(t, y, z)$ satisfies a Nagumo condition with respect to the pair $\alpha(t), \beta(t)$ in case there exists a positive continuous function $\phi(s)$ on $[0, \infty)$ such that $|f(t, y, z)| \leqq \phi(|z|)$ for all $t \in[0,1], \alpha(t) \leqq y \leqq \beta(t)$, $|z|<\infty$, and such that

$$
\int^{\infty} \frac{s}{\phi(s)} d s=+\infty
$$

Received by the editors September 7, 1970.

AMS 1970 subject classifications. Primary 34B15; Secondary 34A40.

Key words and phrases. Boundary value problems, nonlinear boundary conditions, Nagumo condition, funnel, funnel cross-section.

${ }^{1}$ Partially supported by the National Science Foundation under Grant GP-11605 and by the Air Force Office of Scientific Research under AFOSR Grant 1243067. 
If $f(t, y, z)$ satisfies a Nagumo condition, then the following condition holds.

(A) For any $0<t_{0} \leqq 1$ there is $N\left(t_{0}\right)>0$ such that, if $y(t)$ is a solution of (1) on $\left[0, t_{0}\right)$ with $\alpha(t) \leqq y(t) \leqq \beta(t)$ on $\left[0, t_{0}\right)$, then $\left|y^{\prime}(t)\right|$ $\leqq N\left(t_{0}\right)$ on $\left[0, t_{0}\right)$. (See, for example, $[4$, p. 428].)

Condition (A) implies condition (B). A proof of this implication can be given using the Kamke convergence theorem [4, p. 14].

(B) Given any $B_{2} \geqq 0$ and any $t_{0} \in(0,1]$ there exists a positive number $N\left(B_{2}\right)$ such that, for any solution $y(t)$ of (1) with $\left|y^{\prime}(0)\right|$ $\leqq B_{2}$ and $\alpha(t) \leqq y(t) \leqq \beta(t)$ for all $t \in\left[0, t_{0}\right],\left|y^{\prime}(t)\right| \leqq N\left(B_{2}\right)$ for all $t \in\left[0, t_{0}\right]$.

For $\alpha(t), \beta(t) \in C[0,1]$ with $\alpha(t) \leqq \beta(t)$ for all $t \in[0,1]$, define $T=\{(t, y, z)|t \in[0,1], \alpha(t) \leqq y \leqq \beta(t)| z \mid,<\infty\}$.

For $t \in[0,1]$, let $C(t)=\{(t, y, z)|\alpha(t) \leqq y \leqq \beta(t)| z \mid,<\infty\}, S_{\alpha}(t)$ $=\{(t, y, z)|y=\alpha(t)| z \mid,<\infty\}$, and $S_{\beta}(t)=\{(t, y, z)|y=\beta(t)| z \mid,<\infty\}$.

THEOREM 1. Assume that condition (B) holds and that there exist $\alpha(t)$, $\beta(t) \in C^{2}[0,1], \alpha(t)<\beta(t)$, such that $\alpha^{\prime \prime}(t)>f\left(t, \alpha(t), \alpha^{\prime}(t)\right), \beta^{\prime \prime}(t)$ $<f\left(t, \beta(t), \beta^{\prime}(t)\right)$ on $[0,1]$. If $S_{1}$ is a compact connected subset of $C(0)$ which intersects both $\left\{(0, \alpha(0), z) \mid z \leqq \alpha^{\prime}(0)\right\}$ and $\left\{(0, \beta(0), z) \mid z \geqq \beta^{\prime}(0)\right\}$ and if $S_{2}$ is a closed connected subset of $C(1)$ which intersects the line $\{(1, y, z) \mid z=k\}$ for every real $k$, then $B V P(1)-(2)$ has a solution $y(t)$ with $\alpha(t) \leqq y(t) \leqq \beta(t)$ on $[0,1]$.

Proof. Define

$$
\begin{aligned}
\bar{f}\left(t, y, y^{\prime}\right) & =f\left(t, \beta(t), y^{\prime}\right)+(y-\beta(t)), & & \beta(t)<y, \\
& =f\left(t, y, y^{\prime}\right), & & \alpha(t) \leqq y \leqq \beta(t), \\
& =f\left(t, \alpha(t), y^{\prime}\right)+(y-\alpha(t)), & & \alpha(t)>y,
\end{aligned}
$$

for all $t \in[0,1],\left|y^{\prime}\right|<\infty$. It suffices to show that the boundary value problem

$$
y^{\prime \prime}=\bar{f}\left(t, y, y^{\prime}\right)
$$

with boundary conditions (2) has a solution $y(t)$ with $\alpha(t) \leqq y(t) \leqq \beta(t)$ on $[0,1]$ since by the definition of $\bar{f}\left(t, y, y^{\prime}\right), y(t)$ is then a solution of BVP (1)-(2).

Note that any solution $y(t)$ of (5) with $\left(0, y(0), y^{\prime}(0)\right) \in S_{1}$, which satisfies $\left(t, y(t), y^{\prime}(t)\right) \in T$ on $\left[0, t_{0}\right]$ for some $t_{0} \in(0,1)$, and $\left(t_{0}, y\left(t_{0}\right), y^{\prime}\left(t_{0}\right)\right) \in$ bndy $T$ has the property that $\left(t, y(t), y^{\prime}(t)\right) \notin T$ for any $t \in\left(t_{0}, 1\right]$ for which $y(t)$ exists. In addition if $\left(0, y(0), y^{\prime}(0)\right) \in S_{1}$ is such that $y(0)=\beta(0)$ and $y^{\prime}(0) \geqq \beta^{\prime}(0)$ or $y(0)=\alpha(0)$ and $y^{\prime}(0)$ $\leqq \alpha^{\prime}(0)$, then $\left(t, y(t), y^{\prime}(t)\right) \notin T$ for any $t \in(0,1]$ for which $y(t)$ exists. 
For assume $t_{0} \in[0,1),\left(t, y(t), y^{\prime}(t)\right) \in T$ for $t \in\left[0, t_{0}\right]$, and, for definiteness, $y\left(t_{0}\right)=\beta\left(t_{0}\right)$. Then $y^{\prime}\left(t_{0}\right) \geqq \beta^{\prime}\left(t_{0}\right)$ and $\beta(t)<y(t)$ for all $t$ in some right neighborhood of $t_{0}$. If $\beta\left(t_{1}\right)=y\left(t_{1}\right)$ for some $t_{1} \in\left(t_{0}, 1\right]$, then there exists $t_{2} \in\left(t_{0}, t_{1}\right)$ with $y\left(t_{2}\right)>\beta\left(t_{2}\right), y^{\prime}\left(t_{2}\right)=\beta^{\prime}\left(t_{2}\right)$, and $y^{\prime \prime}\left(t_{2}\right) \leqq \beta^{\prime \prime}\left(t_{2}\right)$. However, $\beta^{\prime \prime}\left(t_{2}\right)<f\left(t_{2}, \beta\left(t_{2}\right), \beta^{\prime}\left(t_{2}\right)\right)=\bar{f}\left(t_{2}, \beta\left(t_{2}\right), \beta^{\prime}\left(t_{2}\right)\right)=\bar{f}\left(t_{2}, \beta\left(t_{2}\right), y^{\prime}\left(t_{2}\right)\right)$ $<\bar{f}\left(t_{2}, y\left(t_{2}\right), y^{\prime}\left(t_{2}\right)\right)=y^{\prime \prime}\left(t_{2}\right)$.

The existence of a solution $y(t)$ of BVP (5)-(2), follows from [1, Theorem 3, p. 1061]. Hence, there exists a solution $y(t)$ of (5) satisfying (2) with $\alpha(t) \leqq y(t) \leqq \beta(t)$. By definition of $\bar{f}(t, y, z), y(t)$ is a solution of BVP (1)-(2).

The next theorem is similar to those in [3] and [7]. Because a Nagumo condition is assumed, our proof is simpler.

Theorem 2. Assume $f(t, y, z)$ satisfies a Nagumo condition with respect to $\alpha(t), \beta(t) \in C^{2}[0,1]$ with $\alpha(t) \leqq \beta(t), \alpha^{\prime \prime}(t) \geqq f\left(t, \alpha(t), \alpha^{\prime}(t)\right)$, and $\beta^{\prime \prime}(t) \leqq f\left(t, \beta(t), \beta^{\prime}(t)\right)$ for all $t \in[0,1]$. If $S_{1}$ and $S_{2}$ are as in Theorem 1 , then $B V P(1)-(2)$ has a solution $y(t)$ with $\alpha(t) \leqq y(t) \leqq \beta(t)$ on $[0,1]$.

Proof. Let $\bar{f}(t, y, z)$ be the modification of $f(t, y, z)$ defined by (4). Define the sequences $\left\{\beta_{n}(t)\right\}$ and $\left\{\alpha_{n}(t)\right\}$ by $\beta_{n}(t)=\beta(t)+1 / n$, $\alpha_{n}(t)=\alpha(t)-1 / n$ for all integers $n \geqq 1$ and all $t \in[0,1]$. Note that on $[0,1]$ each pair $\alpha_{n}(t), \beta_{n}(t)$ is such that $\alpha_{n}(t)<\beta_{n}(t), \alpha_{n}^{\prime \prime}(t)>\bar{f}\left(t, \alpha_{n}(t), \alpha_{n}^{\prime}(t)\right)$, and $\beta_{n}^{\prime \prime}(t)<\bar{f}\left(t, \beta_{n}(t), \beta_{n}^{\prime}(t)\right)$.

By assumption, $f\left(t, y, y^{\prime}\right)$ satisfies a Nagumo condition with respect to $\alpha(t)$ and $\beta(t)$. From the definition of $\bar{f}\left(t, y, y^{\prime}\right)$, it follows that $\left|\bar{f}\left(t, y, y^{\prime}\right)\right| \leqq \phi\left(\left|y^{\prime}\right|\right)+1$ for all $t \in[0,1], \alpha_{n}(t) \leqq y \leqq \beta_{n}(t)$, and $\left|y^{\prime}\right|<\infty$ where $\phi$ is the Nagumo function for $f$. It is clear that

$$
\int^{\infty} \frac{s}{\phi(s)+1} d s=+\infty \quad \text { if } \int^{\infty} \frac{s}{\phi(s)} d s=+\infty .
$$

Hence, $\bar{f}\left(t, y, y^{\prime}\right)$ satisfies a Nagumo condition with respect to every pair $\alpha_{n}(t), \beta_{n}(t)$. Choose $\left(0, y_{1}, y_{1}^{\prime}\right) \in S_{1} \cap S_{\beta}(0)$ and $\left(0, y_{2}, y_{2}^{\prime}\right)$ $\in S_{1} \cap S_{\alpha}(0)$ such that $y_{1}^{\prime} \geqq \beta^{\prime}(0)$ and $y_{2}^{\prime} \leqq \alpha^{\prime}(0)$ and define

$$
\begin{gathered}
S_{1}^{*}=S_{1} \cup\left\{\left(0, y, y_{1}^{\prime}\right) \mid \beta(0) \leqq y \leqq \beta(0)+1\right\} \\
\cup\left\{\left(0, y, y_{2}^{\prime}\right) \mid \alpha(0)-1 \leqq y \leqq \alpha(0)\right\} .
\end{gathered}
$$

Since each $\alpha_{n}(t)$ and $\beta_{n}(t)$ satisfies $\alpha_{n}^{\prime \prime}(t)>f\left(t, \alpha_{n}(t), \alpha_{n}^{\prime}(t)\right)$ and $\beta_{n}^{\prime \prime}(t)$ $<f\left(t, \beta_{n}(t), \beta_{n}^{\prime}(t)\right)$, respectively, with $\alpha_{n}(t)<\beta_{n}(t)$ and since $\bar{f}(t, y, z)$ satisfies a Nagumo condition with respect to $\alpha_{n}$ and $\beta_{n}$ (hence, condition (B) relative to $\alpha_{n}(t)$ and $\beta_{n}(t)$ is satisfied), we may evoke Theorem 1 to conclude that $y^{\prime \prime}=\bar{f}\left(t, y, y^{\prime}\right),\left(0, y(0), y^{\prime}(0)\right) \in S_{1}^{*},(1, y(1)$, 
$\left.y^{\prime}(1)\right) \in S_{2}$, has a solution $y_{n}(t)$ for each $n \geqq 1$ with $\alpha_{n}(t) \leqq y_{n}(t) \leqq \beta_{n}(t)$ on $[0,1]$.

By the Arzela-Ascoli Theorem, we conclude that there exists a solution $y(t) \in C^{2}[0,1]$ of BVP (5)-(2) with $\alpha(t) \leqq y(t) \leqq \beta(t)$. But then $y(t)$ is in fact a solution of BVP (1)-(2).

3. Assuming $f(t, y, z)$ satisfies a Nagumo condition with respect to $\alpha(t), \beta(t) \in C^{2}[0,1]$ for $\alpha(t) \leqq \beta(t)$ for all $t \in[0,1]$, let

$\lambda=\max \left(|\alpha(0)-\beta(1)|,|\alpha(1)-\beta(0)|, \max _{[0,1]}\left|\alpha^{\prime}(t)\right|, \max _{[0,1]}\left|\beta^{\prime}(t)\right|\right)$

and define $N(t)$ by

$$
\int_{\lambda}^{N(t)} \frac{s}{\phi(s)} d s=\max _{u \in[0, t]} \beta(u)-\min _{u \in[0, t]} \alpha(u) .
$$

Let

$$
F(x)=\int_{\lambda}^{x} \frac{s}{\phi(s)} d s
$$

then

$$
N(t)=F^{-1}\left(\max _{[0, t]} \beta(u)-\min _{[0, t]} \alpha(u)\right)
$$

is a continuous function on $[0,1]$. Let $N=\min _{[0,1]} N(t)$. Define

$$
\begin{aligned}
S_{3}= & \{(0, y, N) \mid \alpha(0) \leqq y \leqq \beta(0)\} \\
& \cup\left\{\left(0, \beta(0), y^{\prime}\right) \mid \beta^{\prime}(0) \leqq y^{\prime} \leqq N\right\}, \\
S_{4}= & \{(0, y,-N) \mid \alpha(0) \leqq y \leqq \beta(0)\} \\
& \cup\left\{\left(0, \alpha(0), y^{\prime}\right) \mid-N \leqq y^{\prime} \leqq \alpha^{\prime}(0)\right\}, \\
S_{5}= & \{(1, y, N(1)) \mid \alpha(1) \leqq y \leqq \beta(1)\} \\
& \cup\left\{\left(1, \alpha(1), y^{\prime}\right) \mid \alpha^{\prime}(1) \leqq y^{\prime} \leqq N(1)\right\}, \\
S_{6}= & \{(1, y,-N(1)) \mid \alpha(1) \leqq y \leqq \beta(1)\} \\
& \cup\left\{\left(1, \beta(1), y^{\prime}\right) \mid-N(1) \leqq y^{\prime} \leqq \beta^{\prime}(1)\right\} .
\end{aligned}
$$

We can now state and prove our main result which shows the dependence of $S_{1}$ and $S_{2}$ on the a priori bounds.

Theorem 3. Assume $f(t, y, z)$ satisfies a Nagumo condition with respect to $\alpha(t), \beta(t) \in C^{2}[0,1]$ with $\alpha(t) \leqq \beta(t)$ and $\alpha^{\prime \prime}(t) \geqq f\left(t, \alpha(t), \alpha^{\prime}(t)\right)$, $\beta^{\prime \prime}(t) \leqq f\left(t, \beta(t), \beta^{\prime}(t)\right)$ on $[0,1]$. If $S_{1}$ is a closed connected subset of $C(0)$ such that $S_{1} \cap S_{3} \neq \varnothing, S_{1} \cap S_{4} \neq \varnothing$ and if $S_{2}$ is a closed connected subset 
of $C(1)$ such that $S_{2} \cap S_{5} \neq \varnothing$ and $S_{2} \cap S_{6} \neq \varnothing$, then $B V P(1)-(2)$ has a solution $y(t)$ with $\alpha(t) \leqq y(t) \leqq \beta(t)$.

Proof. Choose $\left(0, y_{1}, y_{1}^{\prime}\right) \in S_{1} \cap S_{3},\left(0, y_{2}, y_{2}^{\prime}\right) \in S_{1} \cap S_{4}$ such that both points belong to the same component, $\bar{S}_{1}$, of $S_{1} \cap\{(0, y, z)|| z \mid \leqq N\}$. Choose $\left(0, \beta(0), y_{3}^{\prime}\right) \in S_{\beta}(0)$ as follows. If $y_{1}=\beta(0)$, let $y_{3}^{\prime}=y_{1}^{\prime}$; if $y_{1}<\beta(0)$, choose $y_{3}^{\prime}>N$. Let $L_{1}$ be the line segment joining $\left(0, y_{1}, y_{1}^{\prime}\right)$ to $\left(0, \beta(0), y_{3}^{\prime}\right)$ where, in the case when $y_{1}=\beta(0), L_{1}=\left\{\left(0, y_{1}, y_{1}^{\prime}\right)\right\} \subset \bar{S}_{1}$. In a similar manner, choose $\left(0, \alpha(0), y_{4}^{\prime}\right) \in S_{\alpha}(0)$ by letting $y_{4}^{\prime}=y_{2}^{\prime}$ if $y_{2}=\alpha(0)$ or $y_{4}^{\prime}<-N$ if $y_{2}>\alpha(0)$, and take $L_{2}$ to be the line segment joining $\left(0, y_{2}, y_{2}^{\prime}\right)$ to $\left(0, \alpha(0), y_{4}^{\prime}\right)$. Let $S_{1}^{*}=L_{1} \cup \bar{S}_{1} \cup L_{2}$ and observe that $S_{1}^{*}$ is compact, connected and intersects both $\left\{(0, \alpha(0), z) \mid z \leqq \alpha^{\prime}(0)\right\}$ and $\left\{(0, \beta(0), z) \mid z \geqq \beta^{\prime}(0)\right\}$.

Pick $\left(1, y_{5}, y_{5}^{\prime}\right) \in S_{2} \cap S_{5}$ and $\left(1, y_{6}, y_{6}^{\prime}\right) \in S_{2} \cap S_{6}$ such that both points belong to the same component, $\bar{S}_{2}$, of $S_{2} \cap\{(1, y, z)|| z \mid \leqq N(1)\}$. Let $L_{5}$ and $L_{6}$ be the half-lines given by $\left\{\left(1, y_{5}, z\right) \mid z \geqq y_{5}^{\prime}\right\}$ and $\left\{\left(1, y_{6}, z\right) \mid z \leqq y_{6}^{\prime}\right\}$ and let $S_{2}^{*}=L_{5} \cup \bar{S}_{2} \cup L_{6}$.

Consider the boundary value problem $y^{\prime \prime}=f\left(t, y, y^{\prime}\right)$,

$$
\left(0, y(0), y^{\prime}(0)\right) \in S_{1}^{*}, \quad\left(1, y(1), y^{\prime}(1)\right) \in S_{2}^{*} .
$$

By the construction of $S_{1}^{*}$ and by the assumption on $f(t, y, z)$, the proof of Theorem 2 implies that there is a compact connected subset $C \subset C(1)$ of the funnel cross-section $F\left(1,0, S_{1}^{*}\right)=\bigcup_{s \in S_{1}^{*}} F(1,0, s)$ (where $F(1,0, s)$ denotes the funnel cross-section at $t=1$ of all solutions emanating from $s$ which exist at $t=1$ ) which intersects both $\left\{(1, y, z) \in S_{\beta}(1) \mid z \geqq \beta^{\prime}(1)\right\}$ and $\left\{(1, y, z) \in S_{\alpha}(1) \mid z \leqq \alpha^{\prime}(1)\right\}$. Hence, BVP (1)-(6) has, by Theorem 2 and the Nagumo condition, a solution $y(t)$ with $\alpha(t) \leqq y(t) \leqq \beta(t),\left|y^{\prime}(1)\right| \leqq N(1)$, and in fact $(1, y(1)$, $\left.y^{\prime}(1)\right) \in S_{2}$. From the Nagumo condition, $\left|y^{\prime}(0)\right| \leqq N$ and by the construction of $S_{1}^{*},\left(0, y(0), y^{\prime}(0)\right) \in S_{1}$. Therefore, BVP (1)-(2) has a solution $y(t)$.

4. As an application of these results consider the following boundary value problem previously considered by Markus and Amundson [6]. This problem arises in studying the dynamics of certain chemical reactions and is given by

$$
\begin{gathered}
y^{\prime \prime}=-L_{1} y^{\prime}-L_{2} k(y), \\
y(0)=0, \quad y^{\prime}(1)=-L_{1} y(1) .
\end{gathered}
$$

The positive constants $L_{1}$ and $L_{2}$ depend on various parameters of the physical problem and $k(y)$ is a continuous, nonincreasing function on $[0, c]$ with $k(y)>0$ for $0 \leqq y<c$ and $k(c)=0$. 
Theorem 3 gives the existence of a solution $y(t)$ to BVP (7)-(8) with $0 \leqq y(t) \leqq c$ since $\alpha(t)=0$ and $\beta(t)=c$ are lower and upper solutions, respectively, and a Nagumo condition is clearly satisfied.

\section{REFERENCES}

1. J. Bebernes and R. Wilhelmsen, $A$ techrique for solving two dimensional boundary value problems, SIAM J. Appl. Math. 17 (1969), 1060-1064.

2. - , A general boundary value problem technique, J. Differential Equations 8 (1970), 404-415.

3. - A remark concerning a boundary value problem (submitted).

4. P. Hartman, Ordinary differential equations, Wiley, New York, 1964. MR 30 \#1270.

5. L. Jackson and G. Klaasen, $A$ variation of the topological method of Wazewski, SIAM J. Appl. Math. (to appear).

6. L. Markus and N. Amundson, Nonlinear boundary value problems arising in chemical reactor theory, J. Differential Equations 4 (1968), 102-113. MR 39 \#7201.

7. S. Seqdziwy, On the existence of a solution of a certain nonlinear boundary value problem (submitted).

University of Colorado, Boulder, Colorado 80302 